\title{
Polarization Insensitive and Transparent Frequency Selective Surface for Dual Band GSM Shielding
}

\author{
Yiming Yang, Weiwei Li, Khaled Salama, and Atif Shamim, Senior Member, IEEE
}

\begin{abstract}
This paper proposes a single-layer, dual-band, optically transparent frequency selective surface (FSS) for GSM shielding. The design evolves from a fractal cross dipole to achieve dual band response, polarization insensitivity and wide angle of incidence stability with a miniaturized size. A two-axis symmetric structure helps to provide a stable frequency response for incident waves with different polarization angles up to $60^{\circ}$. Capacitive loading further reduces the unit size and extends the maximum stable incident angle. The working mechanism of the design has been explained through an equivalent lumped element circuit model (ECM), which provides a generic approach to retune or optimize the design for another frequency band. Both the fullwave EM and circuit simulations are in good agreement. A prototype has been realized by screen-printing a custom silver nanowires (Ag NWs) based transparent ink on a flexible polymer substrate. A decent reflection performance has been achieved from 0.71 to $1.25 \mathrm{GHz}$ and from 1.73 to $2.16 \mathrm{GHz}$ in measurements, which are consistent with the simulations. Optical measurements reveal a transparency of $81.6 \%$. Printed FSS is completely flexible and the performance does not deteriorate after bent or rolled up conditions. Field testing on a glass box demonstrate decent shielding from the incoming GSM signals. The highly transparent and flexible nature of the FSS structure makes it suitable for mounting on glass windows of cars or home environments for EM shielding purposes.
\end{abstract}

Index Terms - Frequency selective surface, fractal cross dipole, GSM Shielding, screen printing, silver nanowires

\section{INTRODUCTION}

$\mathrm{W}$ ITH the growing popularity of wireless communication, radio frequency $(\mathrm{RF})$ devices are covering more and more frequencies to cater for either additional bandwidth requirements or new applications [1-10]. Although there are national standards for maximum electromagnetic interference (EMI) and radio power ratings for each device, the large numbers of RF devices working in close proximity suffer from radio interference. Besides, there are increasing concerns about effects of extensive electromagnetic exposure to humans. This is particularly true for vulnerable patients and newborn babies in hospital environments. Therefore, there is considerable interest in selective RF shielding and researchers are striving to

Manuscript received February 18, 2019; (Corresponding author: Weiwei Li) Y. Yang, W. Li, K. Salama, and A. Shamim are with the Computer, Electrical and Mathematical Sciences and Engineering Division, King Abdullah University of Science and Technology, Thuwal 23955-6900, Saudi Arabia. (Email: $\quad$ yiming.yang @kaust.edu.sa; $\quad$ weiwei.li@kaust.edu.sa; khaled.salama@kaust.edu.sa; atif.shamim@kaust.edu.sa) develop new materials and designs to shield the electromagnetic waves. For example, high-performance shielding materials, capable of absorbing the EM wave, have been widely studied in the past few decades [11-14]. However, the absorption of these materials is frequency insensitive, which means that both wanted and unwanted frequencies of EM wave are shielded. Moreover, the absorbed EM waves are converted into heat, which is not conducive for many applications. Frequency selective surface (FSS) reflector, on the contrary, provides selective shielding where unwanted frequency bands are reflected and other bands are allowed to pass through. FSS has been widely used in radar radome design, special filtering, electromagnetic shielding, and many other fields [15-17]. Novel fabrication techniques, such as screen-printing with conductive ink, are also providing FSS with flexibility and transparency for new applications [18-22]. With flexibility and transparency, FSS can provide EM shielding for unconventional mediums and applications. For example, a newborn incubator in a hospital, or a glass window in a car or home can reflect the unwanted EM waves without losing the glass transparency.

To achieve better shielding effectiveness, state-of-the-art FSS design must be polarization-insensitive, provide stable performance under wide angles of incidence, and show multi band or wide band reflection. For other emerging applications, optically transparent and mechanically flexible FSS structures are also highly desirable. Many efforts have been made in the past to design FSS elements that meet the above-mentioned requirements. For example, [23] combines meandering and inter-layer coupling to increase stable incident angle range up to $80^{\circ}$, but the asymmetrical patterns show performance deviation for TE and TM polarizations. In [24], the authors propose a meandered handshake design to reduce the unit size and increase angular stability, but the single layer pattern is only $180^{\circ}$ symmetric, showing different responses for TE and TM polarized waves. The polarization insensitivity has been achieved by adding another layer with $90^{\circ}$ rotated pattern. However, the double layer compact design provides little extra space to achieve multiband operation for the desired frequency bands. In [25], the authors proposes both fractal miniaturization as well as $90^{\circ}$ rotational symmetry for a polarization insensitive compact design, but multi-band operation is not feasible for this

Y. Yang is with School of Electronics Science and Engineering, University of Electronic Science and Technology of China, Chengdu 610054, China

Color versions of one or more of the figures in this paper are available online at http://ieeexplore.ieee.org.

Digital Object Identifier 
single layer design. Higher dimensional structures, i.e., 2.5-D and 3-D, have the ability to achieve dual-band and polarization insensitive performance with a compact size [26, 27]. However, with such designs, fabrication complexity and cost increases considerably and they cannot be used for flexible applications. For flexible designs and low-cost fabrication, additive manufacturing techniques, such as screen-printing, are promising [9, 21, 28-30]. Some papers have reported printed FSS structures [31-33], but none has been realized through a transparent ink. A couple of papers report optically transparent FSS designs based on printed meandered structures [22, 34], where metal pastes with high solid content have been utilized for printing, and the optical transparency has been achieved through the uncovered areas of the designed patterns. This kind of FSSs typically demonstrates low optical transparency due to the opaque nature of the conductive materials. In addition, due to the meandered elements and the relying on the open areas, the design capability of these designs has been highly limited to single band, and it is extremely difficult to achieve multi-band operation with a single layer.

In this paper, we have designed and screen-printed a single layer, dual-band, transparent FSS demonstrating polarization insensitivity as well as a decent range for angular stability. The working mechanism is studied through an ECM. Transparency and flexibility have been achieved through screen-printing of a custom Ag NWs based conductive ink on a flexible polymer substrate. Field testing has been done on a glass box before and after bending the FSS, and good reflection is achieved for both GSM bands, verifying the predicted performance from the full wave as well as ECM simulations.

\section{FSS DESIGN AND ANALYSIS}

\section{A. FSS Design Evolution}

The proposed FSS and its design evolution steps are shown in Fig. 1. The FSS structure is screen-printed on a flexible and transparent polyethylene terephthalate (PET) sheet with a dielectric constant of 2.6, a loss tangent of 0.0025 , and a thickness of $0.05 \mathrm{~mm}$. A custom transparent $\mathrm{Ag} \mathrm{NW}$ ink with an electrical conductivity of $5 \times 10^{5} \mathrm{~S} / \mathrm{m}$ has been used to realize the FSS structure. The transparent conductor has a thickness of $2 \mu \mathrm{m}$. By utilizing the method, the flexible and transparent FSS can be easily bonded to window glass with curved surfaces. The first stage for the design is a second order fractal cross dipole (Fig. 1(a)), which is an attractive option for miniaturized and multi-band FSS design [35]. The structure has two reflective resonant modes and is favorable for dual-band applications because of the self-similar fractal shape. The unit cell has $90^{\circ}$ symmetry, which is beneficial for achieving polarization insensitivity as it offers identical response for both TE and TM modes. In addition, the fractal design reduces the overall size of the unit cell due to the self-meandering shape, and therefore, the stable incident angle range is increased.

To illustrate the aforementioned benefits of the first stage design, the frequency response for TE and TM waves as well as the current distribution for each resonant frequency are plotted in Fig. 2. The simulations have been performed in Ansys HFSS

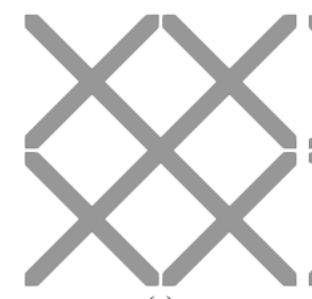

(a)

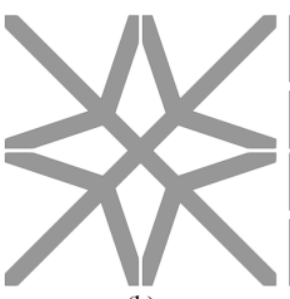

(b)

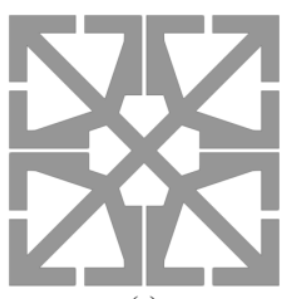

(c)
Fig. 1. Design evolutions of the proposed FSS structure. (a) First design of cross dipole. (b) Design with tunable frequencies. (c) Final structure.

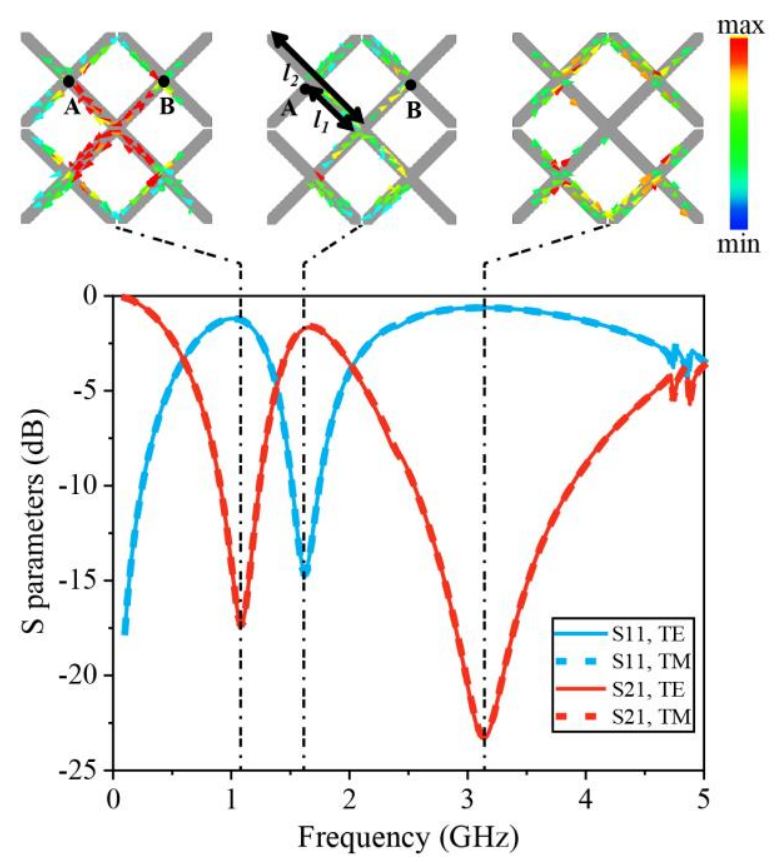

Fig. 2. The simulated S-parameters and modes for the first design stage.

utilizing Floquet port excitation and master/slave boundary configuration. From the plot, we can observe two transmission zeros at $1.09 \mathrm{GHz}$ and $3.13 \mathrm{GHz}$ and a transmission pole occurring at $1.64 \mathrm{GHz}$. The solid line for TE response and the dashed line (not clearly visible in Fig. 2 due to the overlap) for TM response overlap each other, which proves that the $90^{\circ}$ rotation symmetry design provides polarization insensitive response. The current distributions on the cross dipole for all the three operating modes (two transmission zeros and one transmission pole) are extracted and shown in Fig. 2. The intensity and direction of the surface currents are indicated by the color and arrow of the vectors, respectively.

At the first transmission zero $(1.09 \mathrm{GHz})$, the currents are mainly distributed in the central cross dipoles. The in-phase currents are derived from the ends of each branch of the structure and gathers at the branch points (indicated by " $\mathrm{A}$ " and its $90^{\circ}$ symmetrical positions). Then, the currents flow along the central branch in the polarized ' $\mathrm{V}$ ' shaped direction to the center of the unit cell and finally scatter at another branch point. The current distribution for the transmission pole $(1.64 \mathrm{GHz})$ is 
different, where the currents mainly distribute around the upper and lower hole-shaped split-ring-loop of the unit cell. Along the polarization axis, the currents flow through point ' $A$ ' and then towards the unit center, but currents in the mirrored direction pass point ' $\mathrm{B}$ ', resulting in zero total resonant current and wave transmission. For the higher frequency transmission zero (3.13 $\mathrm{GHz}$ ), less current flows in the center of the branching point as compared to the first transmission zero. On the contrary, the currents are distributed at each quarter branch, originating from one side of the tip, gathering at point " $\mathrm{A}$ " and then flowing to another tip.

From the current distributions, we can see that all of the three modes share one junction point in common (point $\mathrm{A}$ and its $90^{\circ}$ symmetry points). Thus, the two reflection frequencies can be tuned by simply moving this point along the diagonal axis, which is the second design stage. Here, we define $\alpha$ as the ratio of the length of the inner branch $\left(l_{1}\right)$ to the complete branch $\left(l_{2}\right)$ (shown in Fig. 2). By decreasing $\alpha$ via moving the branch point closer to the unit center, the current travels a shorter distance for the $1 \mathrm{GHz}$ mode but longer for the $3 \mathrm{GHz}$ mode. Thus, the lower resonant frequency increases and the higher resonant frequency decreases, resulting in a lowered frequency ratio. Similarly, the frequency ratio will increase if the branch point is moved away from the unit center. To verify the tuning ability, EM simulation has been carried out to sweep $\alpha$ from 0.2 to 0.8 . As can be seen in Fig. 3, by increasing $\alpha$, the lower resonant frequency decreases from 1.3 to $0.88 \mathrm{GHz}$, while the higher frequency increases from 2.5 to $3.5 \mathrm{GHz}$, changing the resonant frequency ratio from 1.9 to 4.0 . It should be noted that another transmission zero appears between the existing zeros when $\alpha$ becomes larger than 0.6. This can be attributed to the significant coupling between the fractal branches. Nevertheless, we can estimate and select the desired frequencies for different applications just by choosing the proper $\alpha$. Here, an $\alpha$ with a value of 0.25 has been selected for the final design because the extracted frequency ratio ( 2) is close to the GSM bands (900 and $1800 \mathrm{MHz}$ ).

From the second design iteration, we obtain a FSS structure capable of resonating at 1.3 and $2.6 \mathrm{GHz}$, both of which are larger than the desired GSM bands. Consequently, in the final design stage, shown in Fig. 1(c), we focus on tuning the resonator to the desired frequency of 0.94 and $1.84 \mathrm{GHz}$. The resonant frequency is determined by $f=1 / \sqrt{L C}$, where $\mathrm{L}$ is the equivalent inductor and $\mathrm{C}$ is the equivalent capacitor. To lower the resonant frequency, one solution is to increase $\mathrm{C}$ by adding capacitance load at the end of the dipoles, as shown in Fig. 1(c). To better understand the capacitance distribution, the electric field of the FSS structure with capacitance wires is shown in Fig. 4. For both resonating modes, the electric is mainly distributed in the gap between the wire ends. Thus, both resonant frequencies can be lowered because of the increased capacitance.

Based on the aforementioned design evolutions, the FSS design concept is easy to be applied to other multi-band applications following three steps: 1) tune the value of $\alpha$ in Fig. 3 to shift the resonant frequency ratio to meet the desired bands, 2) change the capacitance stub length and the branch width
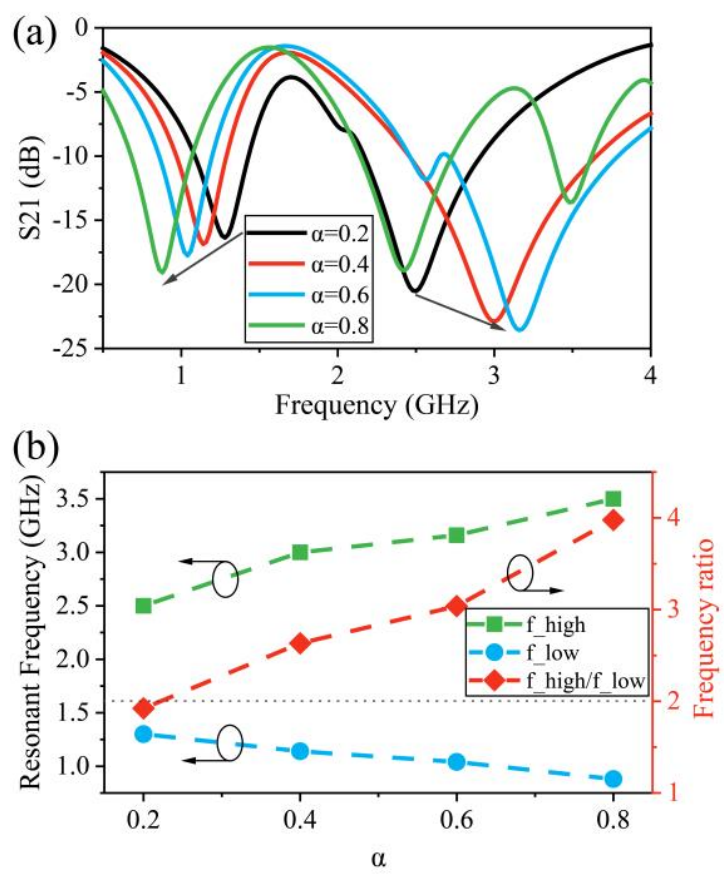

Fig. 3. The (a) simulated $S_{21}$ and (b) the extracted resonant frequency and frequency ratio for the second stage of the design with different $\alpha$.

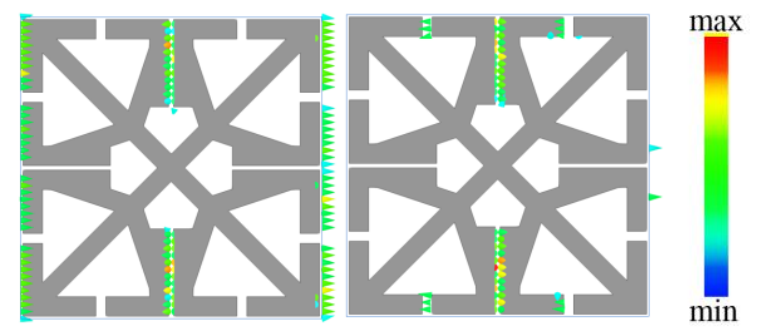

Fig. 4. E-field of the FSS structure with capacitance wires at frequency of $0.94 \mathrm{GHz}$ (left) and $1.84 \mathrm{GHz}$ (right).

further to control the resonant frequencies and the reflection bandwidth, and 3) adjust the overall size of the design to fit the desired resonant frequency.

\section{B. Performance Assessment for Varying Polarization and Angle of Incidence}

By tuning the stub lengths, the detailed dimensions of the final FSS structure, together with a PET substrate and a poly[methyl methacrylate] (PMMA) glass, are shown in Fig. 5(a). The design consists of a large cross dipole along the diagonal direction, 8 branches lying from point $\mathrm{A}$ to the center of the boundaries, and capacitance wires. EM simulation results, shown in Fig. 5(b), confirm that the optimized FSS exhibits two resonance frequencies of $0.94 \mathrm{GHz}$ and $1.84 \mathrm{GHz}$ with a bandwidth of $10 \%$, meeting the requirements for GSM shielding. As compared with the design in stage two, another transmission zero appears at $2.72 \mathrm{GHz}$, which does not interfere with the GSM reflection frequency range. 
(a)
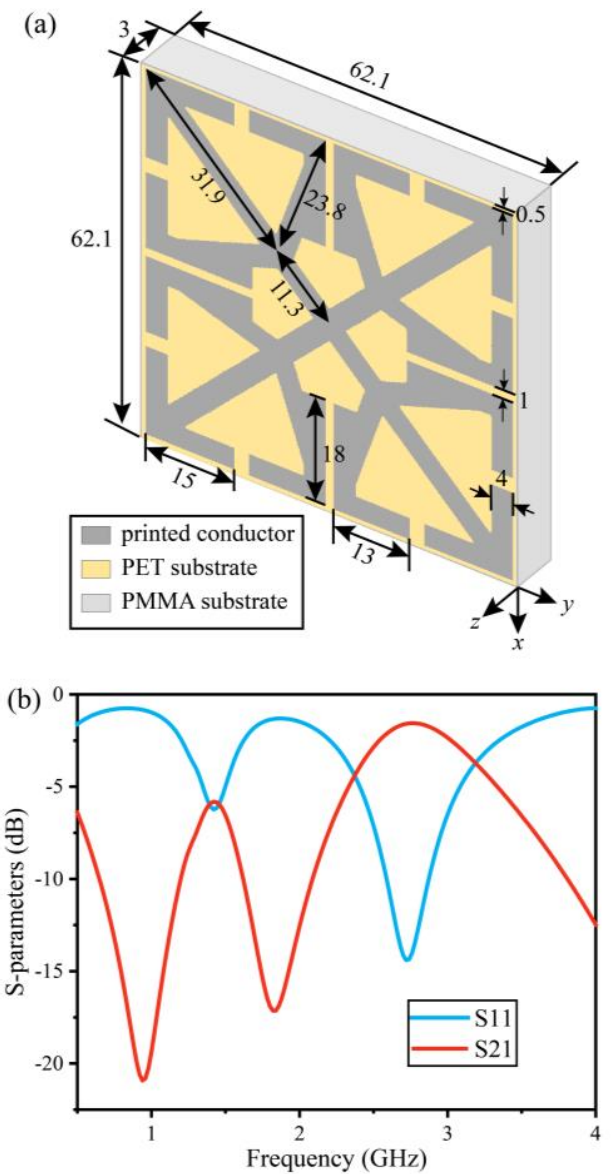

Fig. 5. (a) The geometry parameters and (b) the simulated Sparameters of the proposed FSS structure.
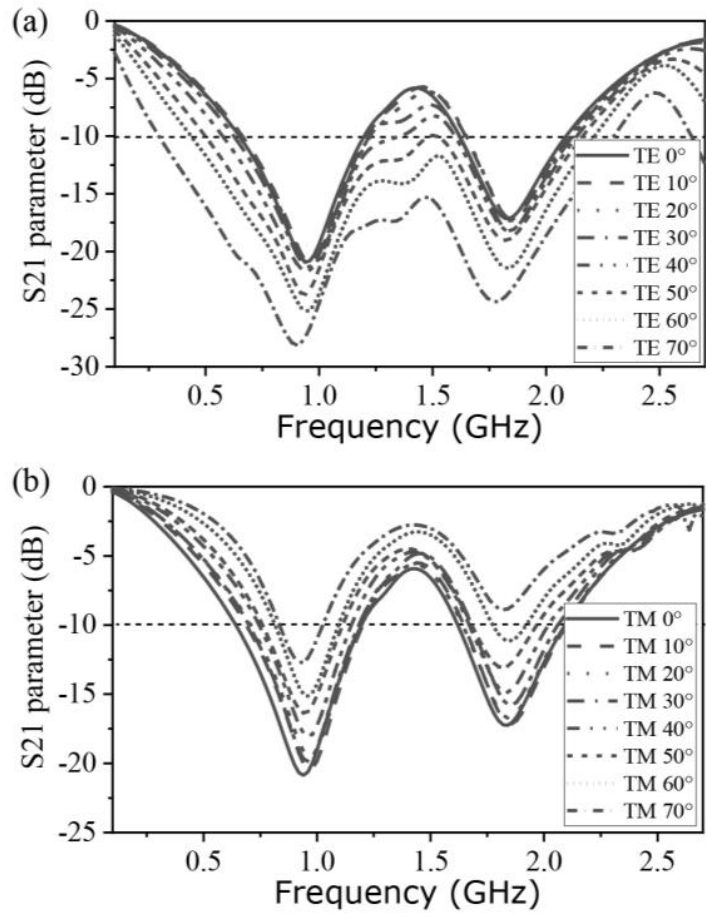

Fig. 6. The simulated S21 of the proposed FSS structure with various incident angles for both TE and TM polarizations.
As stable performance for varying polarization and incident angles is important for FSS structures, we assessed our proposed FSS design for various incident angles ranging from $0^{\circ}$ to $70^{\circ}$ for both TE and TM modes. As shown in the EM simulation results in Fig. 6, the proposed FSS structure shows a stable resonance $\left(\mathrm{S}_{21}\right.$ below $\left.-10 \mathrm{~dB}\right)$ from $0^{\circ}$ to $60^{\circ}$ and from $0^{\circ}$ up to $70^{\circ}$ for $\mathrm{TM}$ and TE polarizations, respectively. Such stability ensures effective GSM shielding for practical applications.

\section{Modal Analysis and Equivalent Circuit Modeling}

To have a deeper understanding of the operation modes, we first estimate the valid frequency range for the fundamental modes before the appearance of the grating lobes. Using spectral expansion method in [36], the fundamental as well as diffractive directions of the transmission and reflection waves can be calculated through equation (1).

$\mathbf{r}_{ \pm}=\hat{\mathbf{a}}_{x}\left(s_{x}+k \frac{\lambda_{g}}{L}\right)+\hat{\mathbf{a}}_{y}\left(s_{y}+n \frac{\lambda_{g}}{L}\right) \pm \hat{\mathbf{a}}_{z} \sqrt{1-\left(s_{x}+k \frac{\lambda_{g}}{L}\right)^{2}-\left(s_{y}+n \frac{\lambda_{g}}{L}\right)^{2}}(1)$

where $\mathbf{r}_{+}$and $\mathbf{r}$ - are the direction vector for the reflected waves and transmission waves, respectively. The coordinate system is the same as displayed in Fig. 5. The incident wave direction vector $s$ for the $\mathrm{x}$ and y components are denoted by $s_{x}$ and $s_{y}$, respectively. In $(1),(k, n)$ is the mode index, where $(0,0)$ represents fundamental modes and other index pairs represents grating lobes. The guided wavelength is denoted by $\lambda_{g}$ and $\mathrm{L}$ is the size of a single unit cell. For the normal incident angle, $s_{x}$ and $s_{y}$ are zero. Therefore, the previous equation can be simplified as (2):

$$
\mathbf{r}_{ \pm}=\hat{\mathbf{a}}_{x} k \frac{\lambda_{g}}{L}+\hat{\mathbf{a}}_{y} n \frac{\lambda_{g}}{L} \pm \hat{\mathbf{a}}_{z} \sqrt{1-\frac{\lambda_{g}^{2}}{L^{2}}\left(k^{2}+n^{2}\right)}
$$

The fundamental modes at normal incidence require $(k, n)$ to be $(0,0)$. Therefore, $r_{ \pm}$equals to $\pm \hat{\mathbf{a}}_{z}$ and both the reflection and the transmission directions are parallel to the $z$-axis. At higher frequencies, however, some other combinations of $\lambda_{g}, k$ and $n$ may lead to a real vector $r_{ \pm}$in (2), resulting in grating lobes. For a given grating index pair $(k, n)$, the critical wavelength $\lambda_{g}$ (maximum wavelength before the appearance of grating lobes) can be calculated by letting $r_{z}$ to be zero. And the critical frequency $f_{c}$ can be obtained from (3) [13].

$$
f_{c}=\frac{c}{\lambda_{c} \sqrt{\frac{1+\varepsilon_{r}}{2}}}
$$

By applying $(0, \pm 1)$ or $( \pm 1,0)$ to $(k, n)$ and use the calculated grating lobe frequency, the valid frequency range for the fundamental modes is estimated from DC to $3.6 \mathrm{GHz}$. We can also calculate and predict that another four grating indexes $( \pm 1, \pm 1)$ will appear around $5.1 \mathrm{GHz}$. Therefore, the simulation range is set from $100 \mathrm{MHz}$ to $5 \mathrm{GHz}$. EM simulation results as well as the current distribution plots are displayed in Fig. 7. 

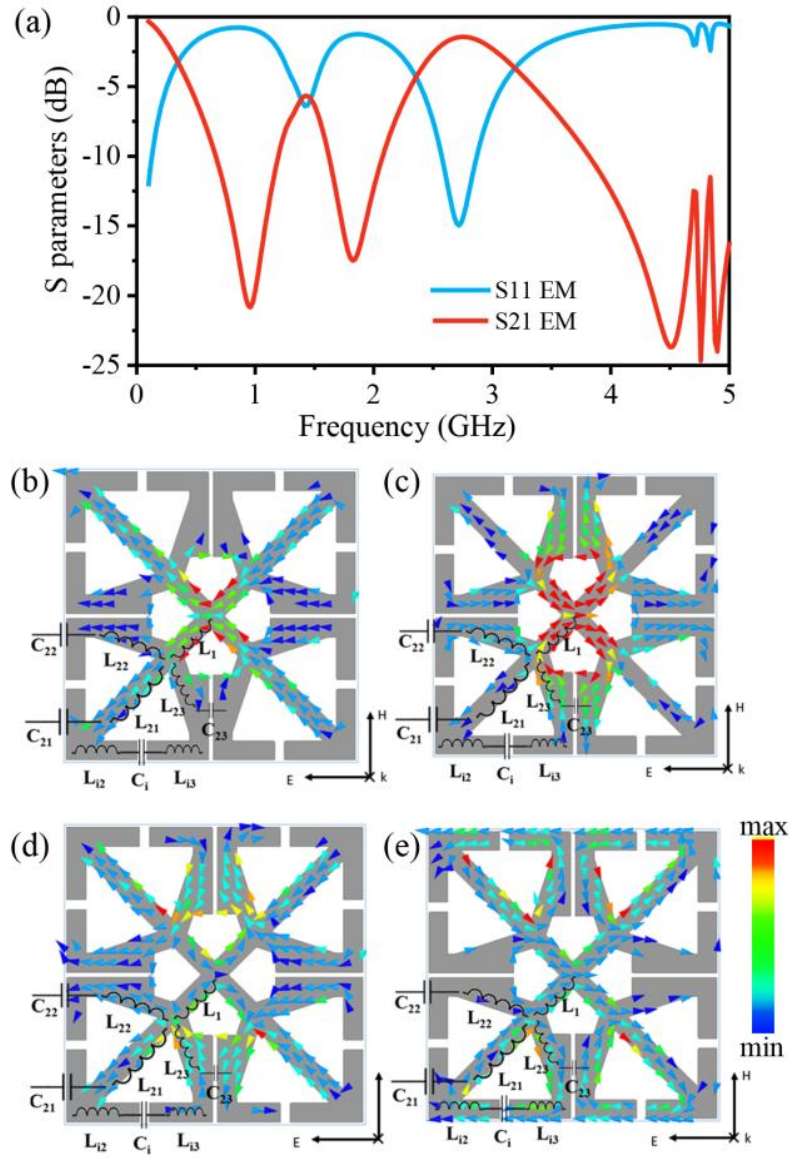

Fig. 7. (a) EM simulation of the final design. The current distributions at (b) $0.94 \mathrm{GHz}$, (c) $1.42 \mathrm{GHz}$, (d) $1.84 \mathrm{GHz}$, and (e) $2.72 \mathrm{GHz}$.

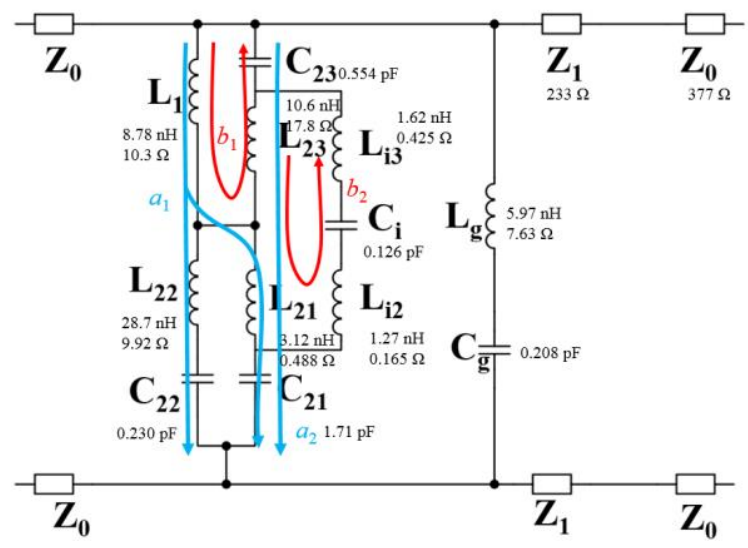

Fig. 8. Equivalent circuit model of the proposed FSS structure.

In Fig. 7(a), we observe transmission zeros at 0.94 and 1.84 $\mathrm{GHz}$ and two transmission poles at 1.42 and $2.72 \mathrm{GHz}$. Grating diffractions appear after $4.5 \mathrm{GHz}$, which is close to our initial estimation. The current distributions at each frequency are shown in Fig. 7(b-e), where the colors and the arrows indicate the intensity and the direction of the current flow, respectively. In Fig. 7(b), the current is mainly distributed along $L_{1}$ and $L_{21}$, and the total current is in parallel with the incident polarized

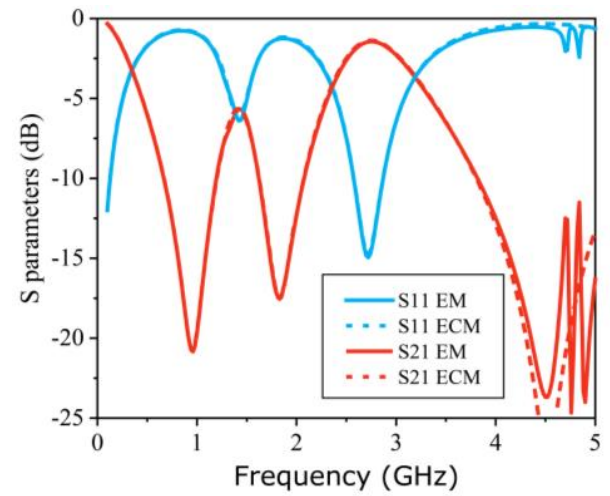

Fig. 9. S-parameters of the proposed FSS structure using EM and ECM simulations.

wave. In Fig. 7(c), the current is distributed along $L_{1}$ and $L_{23}$, which form a closed loop. In Fig. 7(d), the current is distributed along $L_{21}$ and $L_{23}$, while the current in Fig. 7(e) is mainly distributed in $\mathrm{L}_{21}, \mathrm{~L}_{23}, \mathrm{~L}_{\mathrm{i} 1}$, and $\mathrm{L}_{\mathrm{i} 2}$. The currents can be modeled as different combinations of inductors and capacitors [37]. As the four branches of the structure are symmetric in $90^{\circ}$, the current of only one branch has been considered for modeling. The complete circuit network is illustrated in Fig. 8.

In Fig. $8, Z_{0}$ is the wave impedance of air and $Z_{1}$ is the wave impedance of the dielectric substrate. The inductor and capacitor labels are the same as noted in Fig. 7(b-e). At 0.94 $\mathrm{GHz}$, the current is more concentrated in $L_{1}, L_{21}$ and $L_{22}$, which can be modeled as a serial resonance path $a_{1}$ in Fig. 8 with the corresponding inductors and the capacitance $C_{21}$ and $C_{22}$. The low impedance of serial resonance leads to a mismatched impedance and therefore, the FSS shows wave reflection. At $1.42 \mathrm{GHz}$, however, $L_{1}, L_{23}$, and $C_{23}$ form a parallel resonance current curve $b_{1}$ and the circuit model can also form a closed resonance loop, resulting in a high impedance and wave transmission. The current reflection mode in $b_{2}$ at $1.84 \mathrm{GHz}$ mostly involves $L_{22}, L_{23}, C_{22}$, and $C_{23}$, while $L_{22}, L_{23}, L_{i 2}, L_{i 3}$, and $C_{i}$ form the transmission mode in $a_{2}$ at $2.72 \mathrm{GHz}$. Another serial resonance circuit of $L_{g}$ and $C_{g}$ represents the grating diffraction frequency at $4.5 \mathrm{GHz}$.

To obtain the values of each component in the ECM, we have used the curve-fitting optimization with the help of transmission poles and zeros [38] in the Advanced Design System (ADS) simulator from Keysight Technologies [39]. To accommodate for the loss of the conductor, a series resistance has been introduced in the inductor model. In addition, to model the grating reflection at $4.5 \mathrm{GHz}$, a series resonance circuit comprising $L_{g}$ and $C_{g}$ has also been introduced in the equivalent circuit. The relationship between $L_{g}$ and $C_{g}$ has been expressed in (4). With (4), $C_{g}$ can be derived from $L_{g}$, which simplifies the tuning process. During the first iteration of the curve-fitting, the values of $L_{g}$ and other inductors have been randomly selected in the range of 0.01 to $100 \mathrm{nH}$, while the values of the capacitors and the resistors have been chosen in the range of 0.01 to 100 $\mathrm{pF}$ and 0.01 to $100 \Omega$, respectively. The simulated $S_{11}$ and $S_{21}$ values are compared with those of the EM simulation results to estimate the error between the S-parameters. Further tuning of the variables continues in the iterations until the error converges 
(a)

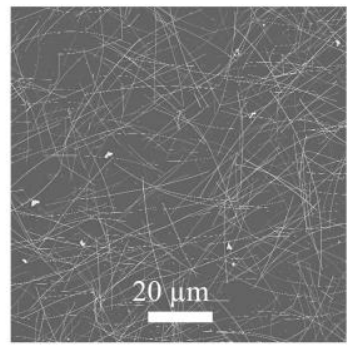

(b)

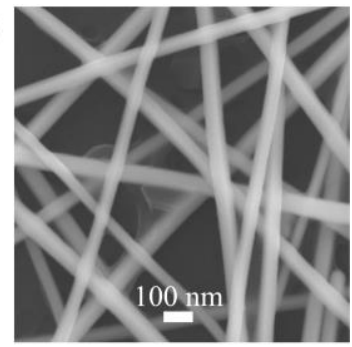

(c)

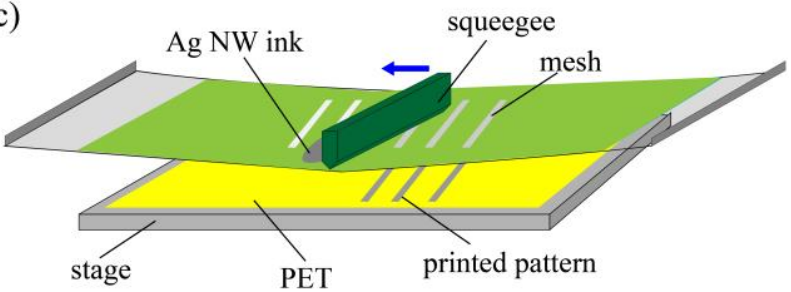

Fig. 10 (a) Scanning electron microscope (SEM) image of the Ag NWs on PET surface. (b) The magnified SEM image of the Ag NWs. (c) The typical screen printing process. (a)

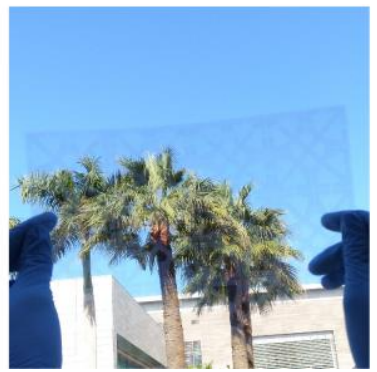

(b)

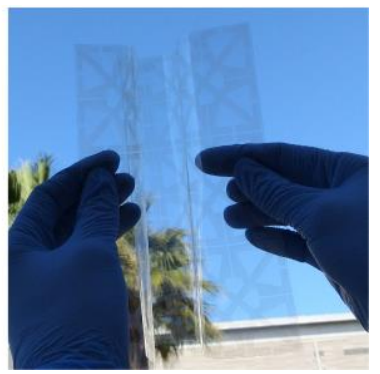

Fig. 11 (a) A digital photograph of the printed FSS. (b) The FSS under bending condition.

to a small value of $0.1 \%$.

$$
C_{g}=\frac{1}{\left(2 \pi f_{c}\right)^{2} L_{g}}
$$

The optimized component values are labeled in Fig. 8. The comparison between the ECM result and the EM simulation of the FSS are displayed in Fig. 9. A very close approximation of these two models within the frequency range of $100 \mathrm{MHz}$ and 3.6 GHz has been observed, validating the estimated frequency range of fundamental modes and the reliability of the proposed model.

\section{FABRICATION AND CHARACTERIZATION}

\section{A. Prototype through Screen Printing}

The proposed FSS has been realized by screen-printing of Ag NWs ink on PET films with a thickness of $50 \mu \mathrm{m}$. The Ag NWs are one-dimensional nanomaterials, exhibiting high optical transmittance as well as high electrical conductivity. In addition, Ag NWs are highly robust and demonstrate excellent stability under mechanical deformations. Thus, the Ag NWs are favorable for flexible and transparent electronics [40, 41].

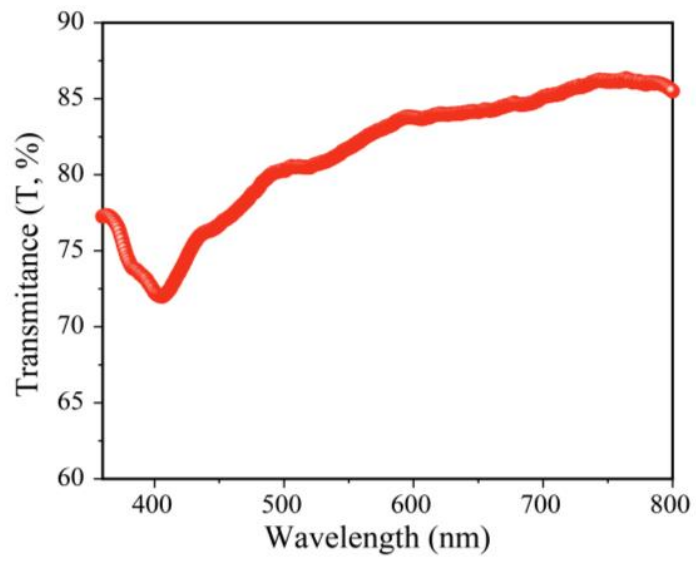

Fig. 12. The optical transmittance spectra of the printed FSS.

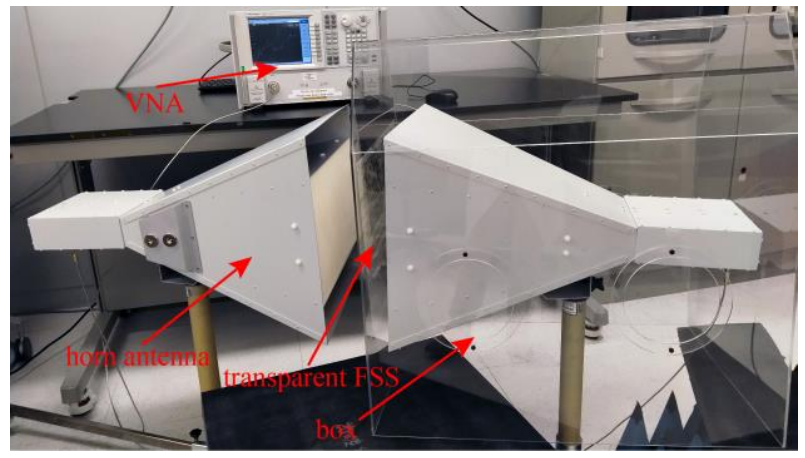

Fig. 13. The glass box with transparent FSS film, together with the measurement setup.

As displayed in Fig. 10(a) and 10(b), the Ag NWs used in this work have an average length of $38 \mu \mathrm{m}$, and an average diameter of $57 \mathrm{~nm}$, demonstrating a length to diameter ratio of more than 600 , which is favorable for high transparency. A custom Ag NW ink suitable for screen printing has been developed for the realization of FSS[42, 43]. Fig. 10(c) illustrates the typical process of the screen-printing, where a rubber squeegee is used to push the Ag NW ink onto PET substrate through open meshes on the screen. A custom stainless-steel screen mesh, containing the optimized FSS structure with an array of $4 \times 3$ unit cells, has been used to screen print the Ag NW ink. The screen mesh has a mesh count of 325 , a mesh angle of $22.5^{\circ}$, and an emulsion thickness of $10 \mu \mathrm{m}$. Screen-printing has been performed on a commercial screen printer (AUREL screen printer 900PA) with a printing speed of $150 \mathrm{~mm} / \mathrm{s}$. After printing, the samples are heated at $100{ }^{\circ} \mathrm{C}$ in an oven for $3 \mathrm{~min}$, followed by immersing in deionized water for $2 \mathrm{~min}$ to remove the polymer binders in the $\mathrm{Ag} \mathrm{NW}$ ink and increase the conductivity. Finally, the printed samples are heated at $120{ }^{\circ} \mathrm{C}$ for 10 min to obtain the flexible FSS with a sheet resistance of about $4 \Omega \mathrm{sq}^{-1}$. Fig. 11(a) shows one fabricated sample with $4 \times$ 3 array elements where the total size is $248 \times 186 \mathrm{~mm}^{2}$. High optical transparency can be observed, as the trees and blue sky can be clearly seen through the FSS. The printed FSS also shows excellent flexibility as depicted in Fig. 11(b).

The optical transparency of the printed FSS has been evaluated by a UV-Vis spectroscopy for the wavelengths of 360 

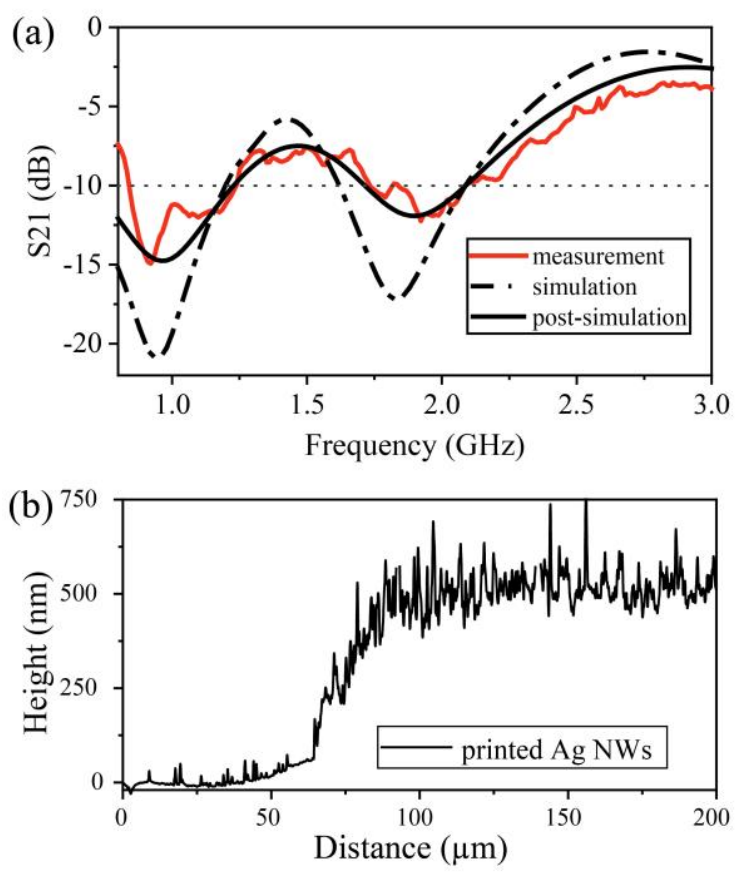

Fig. 14. (a) Experimentally measured and simulated transmission response of the transparent FSS film on a glass box at normal incident angle over 0.8-3 GHz. (b) The measured thickness of the printed Ag NWs layer.

to $800 \mathrm{~nm}$, covering the visible light range. As displayed in Fig. 12 , the printed FSS demonstrates a high transmittance over $80 \%$ for most of the range and a transmittance of $81.6 \%$ at a wavelength of $550 \mathrm{~nm}$. It is noteworthy that the transparency performance is comparable to some exiting commercial products. For example, [44] and [45] report a transparent thin film structure based on commercially available AgHT-4, which has been fabricated through the standard photolithographic etching process. The thin film, utilizing the commercial AgHT4 , exhibits an optical transparency of $70 \%$, which is lower than that of our printed Ag NW FSS (81.6\%), though the sheet resistances are at the same level (about $4 \Omega \mathrm{sq}^{-1}$ ). In addition to the enhanced transparency, our screen-printing technique also has the advantages of easy processing, direct patterning, and large-area and high-volume manufacturing.

\section{B. Experimental Performance Evaluation}

To characterize the frequency response of the printed FSS, two broadband high gain horn antennas have been used and the transmission response of the FSS, while mounted on a glass box, has been recorded by an Agilent N5225A model vector network analyzer (VNA). Before the measurement, a standard open-short-load-through calibration has been performed for $0.8-3 \mathrm{GHz}$ frequency range. As the size of the horn antennas are $350 \times 220 \mathrm{~mm}^{2}$, at least four pieces of transparent FSS (total 8 $\times 6$ arrays) have been bonded to the surface of the glass box to cover the whole antenna and minimize the edge diffraction. The measurement setup are displayed in Fig. 13.

Fig. 14(a) shows the measured $S_{21}$ together with the simulated results. A good agreement in the resonant frequencies and bandwidths can be observed in this comparison figure. The (a)
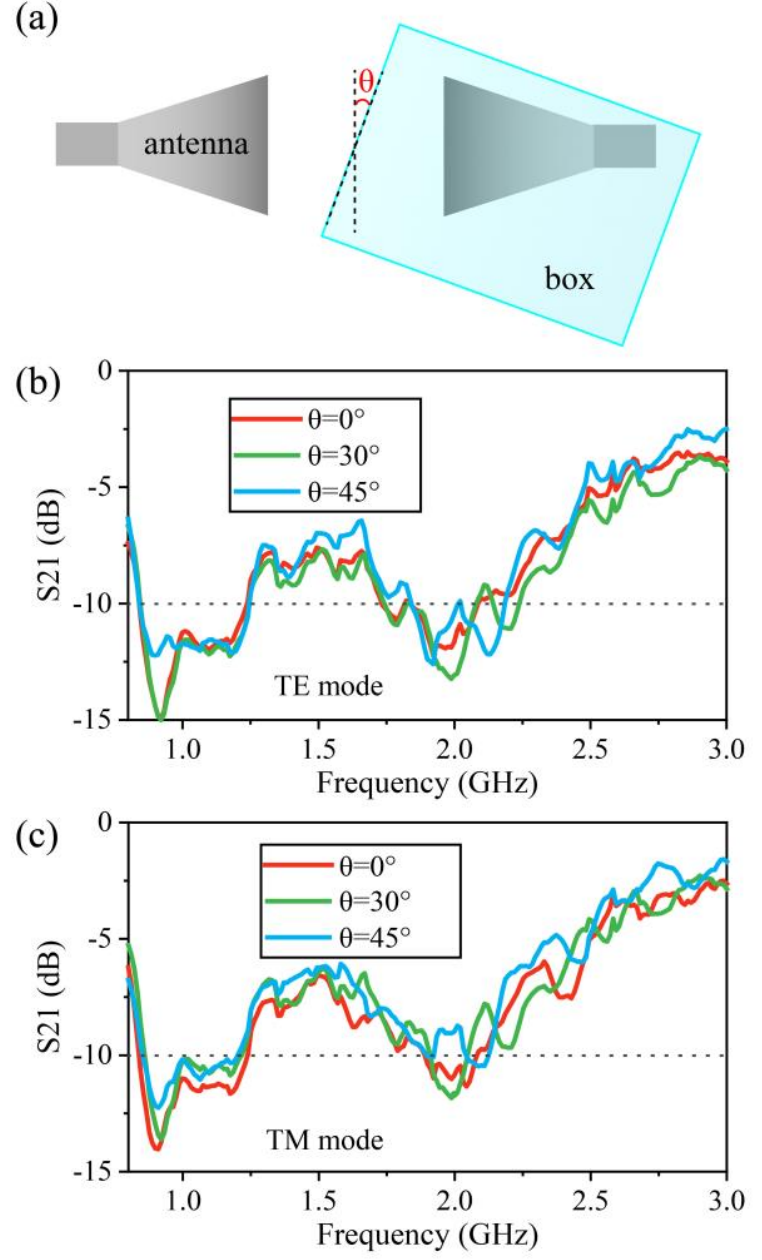

Fig. 15. (a) Diagram of the measurement of the oblique angles (top view). The measured transmission response of the transparent FSS on a glass box at oblique angles of $0^{\circ}, 30^{\circ}$, and $45^{\circ}$ for (b) TE and (c) TM modes over 0.8-3 GHz.

measured transmission response shows two transmission zeros at the frequencies of 0.92 and $1.9 \mathrm{GHz}$ with bandwidths of 0.43 and $0.4 \mathrm{GHz}$, respectively. These results are close to the simulated values of 0.94 and $1.84 \mathrm{GHz}$ with bandwidths of 0.4 and $0.5 \mathrm{GHz}$. However, we observed noticeable differences in the isolation between the measured and the simulated results (i.e., a $7 \mathrm{~dB}$ difference). One of the major reasons for this discrepancy is the difference in the conductor thickness used in the simulations $(2 \mu \mathrm{m})$ versus the thickness of the measured prototype (about $500 \mathrm{~nm}$ ). Our initial plan was to have a conductor thickness of $2 \mu \mathrm{m}$. However, after fabricating a sample with $2 \mu \mathrm{m}$ thickness, we realized that it has a lower optical transparency. Thus, to improve the optical transparency, we fabricated a sample with $500 \mathrm{~nm}$ thickness, which can be observed in Fig. 14(b). This difference in thickness has an effect on the EM properties of the FSS structure. The thin conductor thickness will increase the equivalent resistance and damp the LC resonance circuit, which will lower the peak values of the reflection. To further investigate the reason for the discrepancy, we inspected the fabricated prototype carefully. Considerable surface roughness has been observed on the sample surface. 
(a)
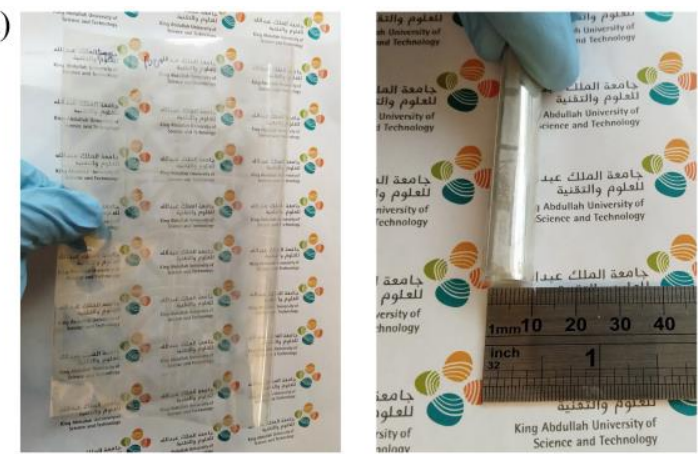

(b)

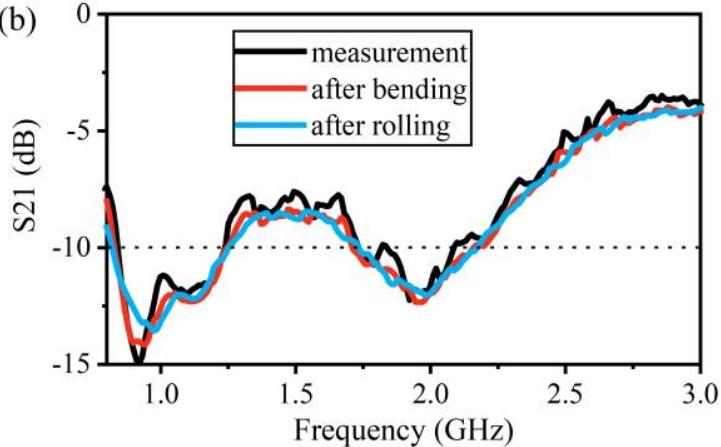

(c)

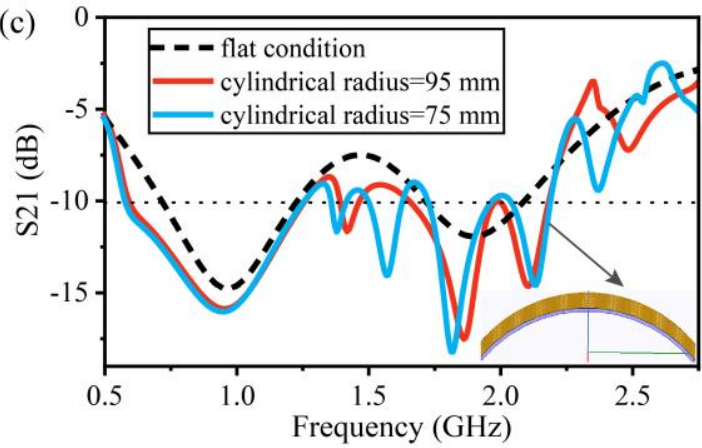

Fig. 16. (a) The printed transparent FSS under bending and rolling conditions. (b) The measured transmission response of the transparent FSS after bending or rolling. (c) The simulated $\mathrm{S}_{21}$ of the FSSs on a cylinder.

This can be ascribed to the pores between the Ag NWs, as has been observed in Fig. 10(b). These two issues are then reinvestigated in the post-measurement simulations by incorporating a thickness of $500 \mathrm{~nm}$ for the conductor, as well as a surface roughness of $0.1 \mu \mathrm{m}$. From Fig. 14(a), we can observe that the post-measurement simulations match well with the measured results. Actually, before running the postmeasurement simulations, the average error between the simulations and measurements is $31 \%$ (over the entire frequency band of interest). After incorporating more accurate values from the fabricated prototype, the average error between the post-measurement simulation results and the measured results is $8.7 \%$. Thus, the simulation model has improved considerably and can predict well the measured results within an error of $8.7 \%$.

We have studied the influence of different incident angles on the response of the transparent FSS for TE and TM modes. The box has been rotated from parallel to the two horns (denoted by $0^{\circ}$ ) to angles of $30^{\circ}$ and $45^{\circ}$, as has been depicted in Fig. 15(a). The measured transmission response for both polarizations at incident angles of $0^{\circ}, 30^{\circ}$, and $45^{\circ}$ are shown in Fig. 15(b) and 15(c). We observe that the resonant frequencies are well centered at 0.92 and $1.9 \mathrm{GHz}$ for all the angles, whereas the transmission isolation slightly increased for higher angles, such as $45^{\circ}$.

We have also investigated the transmission response of the printed FSS after it has been either bent or rolled, as shown in Fig. 16(a). The bending and rolling have been done after detachment of the printed films from the box. After the recovery to flat condition, the films have been attached to the box again for another measurement. As displayed in Fig. 16(b), negligible changes have been observed in the measured S21 after bending the printed films or rolling with radii down to $5 \mathrm{~mm}$. In addition to the attachment to the flat surface, the FSSs have also been attached to the conformal surface, such as a cylinder, and the $\mathrm{S}_{21}$ has been studied. As shown in Fig. 16(c), despite the fluctuation at some frequencies, the simulated $\mathrm{S}_{21}$ of the FSSs on a cylinder with a radius of 95 and $75 \mathrm{~mm}$ are similar to the response in the flat condition. The isolations at 0.94 and 1.9 $\mathrm{GHz}$ for the FSSs with a cylinder radius of $75 \mathrm{~mm}$ are -15.9 and $-12 \mathrm{~dB}$, respectively. These results are close to the values of 14.8 and $-11.9 \mathrm{~dB}$ for the FSSs in the flat condition, which validates the flexibility and stability of the transparent FSS structures.

We have also compared our printed FSS structures with transparent FSSs in the literature. Many reported works do not use inherently transparent materials. The transparency has been achieved by etching the material (mostly opaque copper sheets) and thus create a mesh structure [46-49]. Such works have relatively higher isolation but lower transparency levels. Furthermore, the reported fabrication processes contain multiple steps, such as coating, laminating, and chemical etching, which are time consuming and costly. It is worth mentioning here that the FSS structure in [46] reports a transparency of $86 \%$, which is a bit misleading as it has been calculated by dividing the empty area of a unit cell to the complete unit cell size. In reality, the optical visibility is not as good as our printed Ag NWs, because pure copper sheet has been used. Although the FSSs reported in [22] and [34] are printed, the opaque silver inks used in the printing process make them unsuitable for applications requiring higher optical transparency. Also, it is extremely difficult to modify the FSS designs reported in [22] and [34] for multi-band operations. In Table I, we have compared all the closely related transparent FSS works with our proposed transparent FSS design (all works owe the transparency to the material property and not to the etched mesh structures or opaque Ag paste). Our printed FSS exhibits higher optical transparency. Some of the designs in the table have neither reported nor studied the sensitivity to polarization and angle of incident wave. Most of the FSS structures are larger in unit cell size than that of our design. Furthermore, they all utilize subtractive techniques, such as etching or milling, to realize the FSS structures, which are time consuming and a waste of materials. More importantly, they are all rigid in nature without any mechanical flexibility. Though, our isolation is relatively low as compared to the etched mesh FSS structures, it is comparable or better than the isolation 


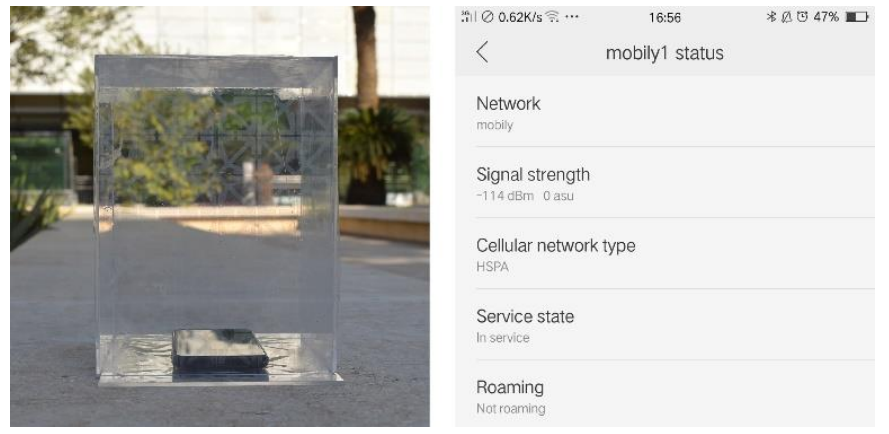

Fig. 17. A shielding box to measure the signal power of a smart phone.

values reported in Table I. In fact, there is a tradeoff between the isolation and the optical transparency. Thicker FSS layer leads to lower transparency, which in turn results in higher isolation. Our simulations demonstrate an isolation of $-22 \mathrm{~dB}$ for a conductor thickness of $2 \mu \mathrm{m}$, however, with a reduced transparency of $60 \%$. It can be concluded from Table I that our design and the printed FSS structures have dual band operation in single layer, excellent transparency, high level of polarization insensitivity and angular stability, and good rejection of EM signals while maintaining mechanical flexibility.

\section{Shielding Effectiveness Demonstration}

To further verify the shielding effectiveness of the printed transparent FSS at GSM bands, we conducted a straightforward EM shielding experiment by placing a smart phone in a shielding box, which has been covered with the printed transparent FSS from all directions. As shown in Fig. 17, six pieces of transparent FSS films are attached on the surface of a PMMA box. The received signal power by the mobile phone, indicated in $\mathrm{dBm}$ unit, is directly recorded from the display screen of the smart phone. For comparison, the experiment is conducted inside as well as outside the PMMA box. For the experiments with box, signals strength is recorded at three different locations, indoor, corridor, and outdoor. As listed in the Table II, the received GSM signal indoor changes from -102 $\mathrm{dBm}$ to $-114 \mathrm{dBm}$ after placing the smart phone into the shielding box, indicating a drop of $12 \mathrm{dBm}$, which is close to the measured transmission attenuation, as has been shown in Fig. 14. Similarly, attenuations in signal power have been observed for other locations as well. The shielding performance of the proposed transparent FSS can be further improved by increasing the thickness, surface uniformity, and electrical conductivity of the Ag NWs.

\section{CONCLUSION}

This work proposes an optically transparent and mechanically flexible FSS structure that can provide dual band shielding from GSM signals. The design is polarization insensitive, and provides stable performance for a wide range incident angles. Prototype has been realized through screenprinting of a custom Ag NW ink on a flexible PET substrate. Decent transparency and EM performance has been achieved in measurements. The prototype works equally well in both planar and bent states, verifying the designs utility for shielding applications requiring conformal designs.

TABLE I

Comparision of the Proposed FSS Structure with Transparent FSSs in the Literature

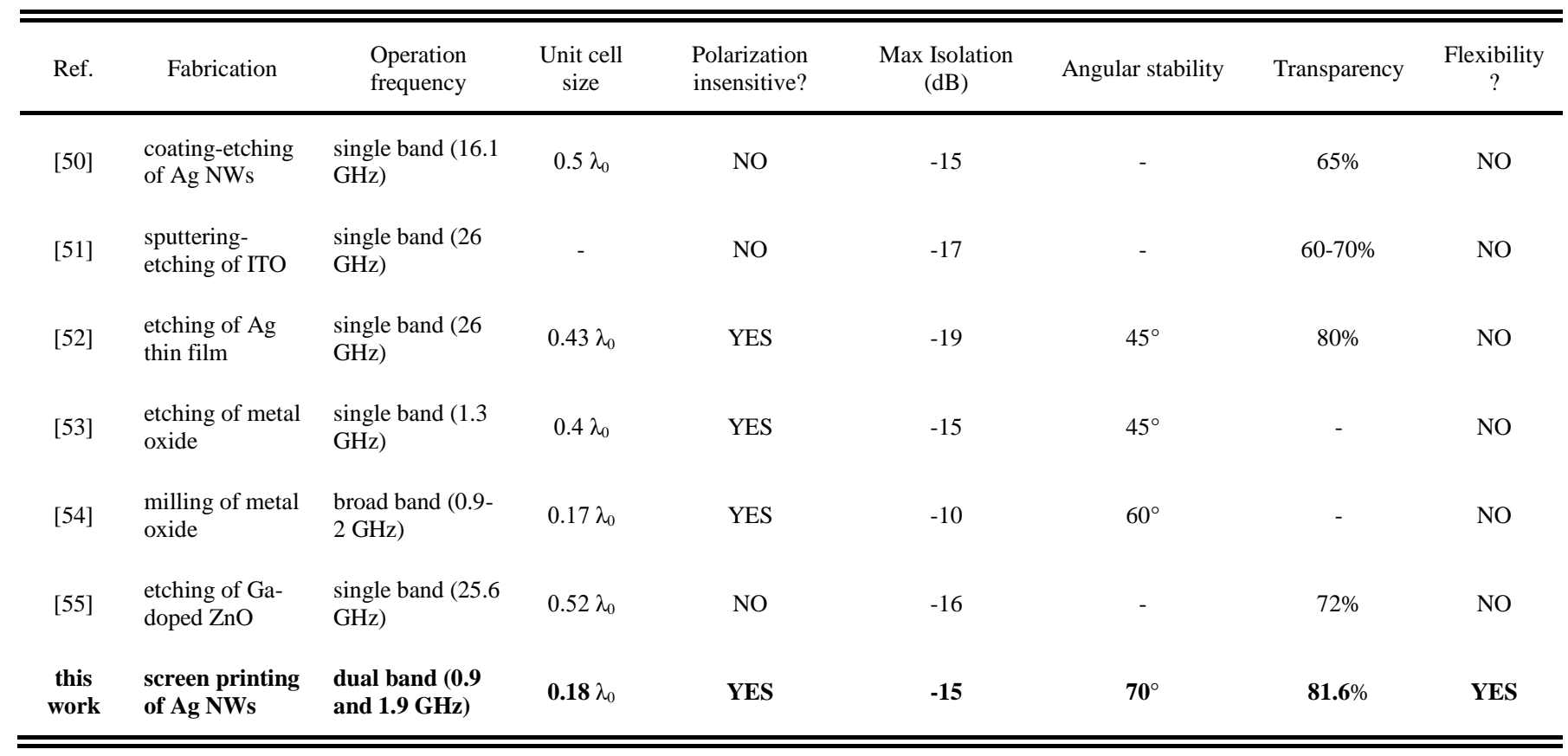


TABLE II

RECEIVED SIGNAL POWER OF THE SMART PHONE IN GSM BANDS

\begin{tabular}{ccc}
\hline \hline Test conditions & Outside the box $(\mathrm{dBm})$ & Inside the box $(\mathrm{dBm})$ \\
Indoor & -102 & -114 \\
Corridor & -94 & -107 \\
Outdoor & -74 & -86 \\
\hline \hline
\end{tabular}

\section{REFERENCES}

[1] R. Hussain, M. S. Sharawi, and A. Shamim, "4-Element Concentric Pentagonal Slot-Line-Based Ultra-Wide Tuning Frequency Reconfigurable MIMO Antenna System," IEEE Trans. Antennas Propag., vol. 66, no. 8, pp. 4282-4287, 2018.

[2] M. Ikram, M. S. Sharawi, and A. Shamim, "A novel very wideband integrated antenna system for 4G and 5G mm-wave applications," Microwave Opt. Technol. Lett., vol. 59, no. 12, pp. 3082-3088, 2017.

[3] G. R. Aiello and G. D. Rogerson, "Ultra-wideband wireless systems," IEEE Microwave Mag., vol. 4, no. 2, pp. 36-47, 2003.

[4] A. Meredov, K. Klionovski, and A. Shamim, "Screen-Printed, Flexible, Parasitic Beam-Switching Millimeter-Wave Antenna Array for Wearable Applications," IEEE Open Journal of Antennas and Propagation, vol. 1, pp. 2-10, 2020.

[5] S. M. Saeed, C. A. Balanis, and C. R. Birtcher, "Inkjet-Printed Flexible Reconfigurable Antenna for Conformal WLAN/WiMAX Wireless Devices," IEEE Antennas Wirel. Propag. Lett., vol. 15, pp. 1979-1982, 2016.

[6] S. Ahmed, F. A. Tahir, A. Shamim, and H. M. Cheema, "A Compact Kapton-Based Inkjet-Printed Multiband Antenna for Flexible Wireless Devices," IEEE Antennas Wirel. Propag. Lett., vol. 14, pp. 1802-1805, 2015

[7] A. Shamim, L. Roy, N. Fong, and N. G. Tarr, "24 GHz On-Chip Antennas and Balun on Bulk Si for Air Transmission," IEEE Trans. Antennas Propag., vol. 56, no. 2, pp. 303-311, 2008.

[8] K. Klionovski, M. S. Sharawi, and A. Shamim, "A DualPolarization-Switched Beam Patch Antenna Array for MillimeterWave Applications," IEEE Trans. Antennas Propag., vol. 67, no. 5, pp. 3510-3515, 2019.

[9] S. Kim, A. Shamim, A. Georgiadis, H. Aubert, and M. M. Tentzeris, "Fabrication of fully inkjet-printed vias and SIW structures on thick polymer substrates," IEEE Trans. Compon. Packag. Manuf. Technol., vol. 6, no. 3, pp. 486-496, 2016.

[10] A. Quddious et al., "Disposable, paper-based, inkjet-printed humidity and $\mathrm{H} 2 \mathrm{~S}$ gas sensor for passive sensing applications," Sensors, vol. 16, no. 12, p. 2073, 2016.

[11] K. Singh et al., "Nanostructured graphene/Fe3O4 incorporated polyaniline as a high performance shield against electromagnetic pollution," Nanoscale, 10.1039/C3NR33962A vol. 5, no. 6, pp. 2411-2420, 2013

[12] S. Kwon, R. Ma, U. Kim, H. R. Choi, and S. Baik, "Flexible electromagnetic interference shields made of silver flakes, carbon nanotubes and nitrile butadiene rubber," Carbon, vol. 68, pp. 118$124,2014 / 03 / 01 / 2014$

[13] C. Luo, T. Jiao, J. Gu, Y. Tang, and J. Kong, "Graphene Shield by SiBCN Ceramic: A Promising High-Temperature Electromagnetic Wave-Absorbing Material with Oxidation Resistance," ACS Appl. Mater. Interfaces, vol. 10, no. 45, pp. 39307-39318, 2018/11/14 2018.

[14] Z. Zeng, H. Jin, M. Chen, W. Li, L. Zhou, and Z. Zhang, "Lightweight and anisotropic porous MWCNT/WPU composites for ultrahigh performance electromagnetic interference shielding," Adv. Funct. Mater., vol. 26, no. 2, pp. 303-310, 2016.

[15] H. Chen, X. Hou, and L. Deng, "Design of Frequency-Selective Surfaces Radome for a Planar Slotted Waveguide Antenna," IEEE Antennas Wirel. Propag. Lett., vol. 8, pp. 1231-1233, 2009.
[16]

I. S. Syed, Y. Ranga, L. Matekovits, K. P. Esselle, and S. Hay, "A Single-Layer Frequency-Selective Surface for Ultrawideband Electromagnetic Shielding," IEEE Trans. Electromagn. Compat., vol. 56, no. 6, pp. 1404-1411, 2014.

[17] M. Bashiri, C. Ghobadi, J. Nourinia, and M. Majidzadeh, "WiMAX, WLAN, and X-Band Filtering Mechanism: Simple-Structured Triple-Band Frequency Selective Surface," IEEE Antennas Wirel. Propag. Lett., vol. 16, pp. 3245-3248, 2017.

[18] W. Y. Yong et al., "Flexible Convoluted Ring Shaped FSS for XBand Screening Application," IEEE Access, vol. 6, pp. 1165711665, 2018.

[19] Y. Tayde, M. Saikia, K. V. Srivastava, and S. A. Ramakrishna, "Polarization-Insensitive Broadband Multilayered Absorber Using Screen Printed Patterns of Resistive Ink," IEEE Antennas Wirel. Propag. Lett., vol. 17, no. 12, pp. 2489-2493, 2018.

[20] W. G. Whittow, Y. Li, R. Torah, K. Yang, S. Beeby, and J. Tudor, "Printed frequency selective surfaces on textiles," Electron. Lett., vol. 50, no. 13, pp. 916-917, 2014.

[21] L. B. Wang, K. Y. See, J. W. Zhang, B. Salam, and A. C. W. Lu, "Ultrathin and Flexible Screen-Printed Metasurfaces for EMI Shielding Applications," IEEE Trans. Electromagn. Compat., vol. 53, no. 3, pp. 700-705, 2011.

[22] A. A. Dewani, S. G. O'Keefe, D. V. Thiel, and A. Galehdar, "Window RF Shielding Film Using Printed FSS," IEEE Trans. Antennas Propag., vol. 66, no. 2, pp. 790-796, 2018.

[23] S. N. Azemi, K. Ghorbani, and W. S. T. Rowe, "Angularly Stable Frequency Selective Surface With Miniaturized Unit Cell," IEEE Microwave Wireless Compon. Lett., vol. 25, no. 7, pp. 454-456, 2015.

[24] T. Hong, K. Peng, and M. Wang, "Miniaturized Frequency Selective Surface Using Handshake Convoluted Stripe," IEEE Antennas Wirel. Propag. Lett., vol. 18, no. 10, pp. 2026-2030, 2019.

[25] Z. Zhao, H. Shi, J. Guo, W. Li, and A. Zhang, "Stopband Frequency Selective Surface With Ultra-Large Angle of Incidence," IEEE Antennas Wirel. Propag. Lett., vol. 16, pp. 553-556, 2017.

[26] W. Yin, H. Zhang, T. Zhong, and X. Min, "A Novel Compact DualBand Frequency Selective Surface for GSM Shielding by Utilizing a 2.5-Dimensional Structure," IEEE Trans. Electromagn. Compat., vol. 60, no. 6, pp. 2057-2060, 2018.

[27] M. W. Niaz, Y. Yin, S. Zheng, L. Zhao, and J. Chen, "Design and Analysis of an Ultra-Miniaturized FSS using 2.5D Convoluted Square Spirals," IEEE Trans. Antennas Propag., pp. 1-1, 2019.

[28] M. F. Farooqui, C. Claudel, and A. Shamim, "An Inkjet-Printed Buoyant 3-D Lagrangian Sensor for Real-Time Flood Monitoring," IEEE Trans. Antennas Propag., vol. 62, no. 6, pp. 3354-3359, 2014. [29] M. Sazegar et al., "Beam Steering Transmitarray Using Tunable Frequency Selective Surface With Integrated Ferroelectric Varactors," IEEE Trans. Antennas Propag., vol. 60, no. 12, pp. 5690-5699, 2012.

[30] Z. Su, K. Klionovski, R. M. Bilal, and A. Shamim, "A Dual Band Additively Manufactured 3-D Antenna on Package With NearIsotropic Radiation Pattern," IEEE Trans. Antennas Propag., vol. 66, no. 7, pp. 3295-3305, 2018.

[31] W. Li, M. Chen, Z. Zeng, H. Jin, Y. Pei, and Z. Zhang, "Broadband composite radar absorbing structures with resistive frequency selective surface: Optimal design, manufacturing and characterization," Compos. Sci. Technol., vol. 145, pp. 10-14, 2017. B. M. Turki et al., "Significant Factors in the Inkjet Manufacture of Frequency-Selective Surfaces," IEEE Trans. Compon. Packag. Manuf. Technol., vol. 6, no. 6, pp. 933-940, 2016.

[33] M. Haghzadeh and A. Akyurtlu, "All-printed, flexible, reconfigurable frequency selective surfaces," J. Appl. Phys., vol. 120, no. 18, p. 184901, 2016.

[34] A. A. Dewani, S. G. O'Keefe, D. V. Thiel, and A. Galehdar, "Optically transparent frequency selective surfaces on flexible thin plastic substrates," AIP Adv., vol. 5, no. 2, p. 027107, 2015.

[35] D. H. Werner and D. Lee, "Design of dual-polarised multiband frequency selective surfaces using fractal elements," Electron. Lett., vol. 36, no. 6, pp. 487-488, 2000.

[36] B. A. Munk, "Spectral Expansion of One- and Two-Dimensional Periodic Structures," in Frequency Selective Surfaces: Theory and Design: John Wiley \& Sons, ch. 4, p. 90.

[37] F. Costa, A. Monorchio, and G. Manara, "An Overview of Equivalent Circuit Modeling Techniques of Frequency Selective 
Surfaces and Metasurfaces," Applied Computational Electromagnetics Society Journal, vol. 29, 12/01 2014.

[38] F. Costa, A. Monorchio, and G. Manara, "Efficient Analysis of Frequency-Selective Surfaces by a Simple Equivalent-Circuit Model," IEEE Antennas Propag. Mag., vol. 54, no. 4, pp. 35-48, 2012.

[39] A. Ghaneizadeh, M. Joodaki, J. Börcsök, A. Golmakani, and K. Mafinezhad, "Analysis, Design, and Implementation of a New Extremely Ultrathin 2-D-Isotropic Flexible Energy Harvester Using Symmetric Patch FSS," IEEE Trans. Microwave Theory Tech., vol. 68, no. 6, pp. 2108-2115, 2020.

[40] L. Hu, H. S. Kim, J.-Y. Lee, P. Peumans, and Y. Cui, "Scalable Coating and Properties of Transparent, Flexible, Silver Nanowire Electrodes," ACS Nano, vol. 4, no. 5, pp. 2955-2963, 2010/05/25 2010.

[41] W. Li, A. Meredov, and A. Shamim, "Coat-and-print patterning of silver nanowires for flexible and transparent electronics," $n p j$ Flexible Electronics, vol. 3, no. 1, p. 19, 2019/12/10 2019.

[42] W. Li, S. Yang, and A. Shamim, "Screen printing of silver nanowires: balancing conductivity with transparency while maintaining flexibility and stretchability," npj Flexible Electronics, vol. 3, no. 1, pp. 1-8, 2019.

[43] W. Li, E. Yarali, A. Bakytbekov, T. D. Anthopoulos, and A. Shamim, "Highly transparent and conductive electrodes enabled by scalable printing-and-sintering of silver nanowires," Nanotechnology, vol. 31, no. 39, p. 395201, 2020.

[44] H. J. Song, T. Y. Hsu, D. F. Sievenpiper, T. J. Talty, and H.-p. Hsu, "Transparent thin film antenna," ed: Google Patents, 2007.

[45] H. J. Song, T. Y. Hsu, D. F. Sievenpiper, H. P. Hsu, J. Schaffner, and E. Yasan, "A method for improving the efficiency of transparent film antennas," IEEE Antennas Wirel. Propag. Lett., vol. 7, pp. 753756, 2008.

[46] U. Farooq, M. F. Shafique, and M. J. Mughal, "Polarization Insensitive Dual Band Frequency Selective Surface for RF Shielding Through Glass Windows," IEEE Trans. Electromagn. Compat., pp. 1-8, 2019.

[47] S. K. Sharma, D. Zhou, A. Luttgen, and C. D. Sarris, "A Micro Copper Mesh-Based Optically Transparent Triple-Band Frequency Selective Surface," IEEE Antennas Wirel. Propag. Lett., vol. 18, no. 1, pp. 202-206, 2019.

[48] S. Can, K. Y. Kapusuz, and A. E. Yilmaz, "Optically transparent frequency selective surface for ultrawideband applications," Microwave Opt. Technol. Lett., vol. 59, no. 12, pp. 3197-3201, 2017.

[49] M. Mantash, A. Kesavan, and T. A. Denidni, "Highly Transparent Frequency Selective Surface Based on Electrotextiles for On-Chip Applications," IEEE Antennas Wirel. Propag. Lett., vol. 18, no. 11, pp. 2351-2354, 2019.

[50] H. J. Song, J. H. Schaffner, K. A. Son, and J. S. Moon, "Optically transparent Ku-band silver nanowire frequency selective surface on glass substrate," in 2014 IEEE Antennas and Propagation Society International Symposium (APSURSI), 2014: IEEE, pp. 2100-2101.

[51] C. Tsakonas et al., "Optically transparent frequency selective window for microwave applications," Electron. Lett., vol. 37, no. 24, pp. 1464-1466, 2001.

[52] E. A. Parker, C. Antonopoulos, and N. E. Simpson, "Microwave band FSS in optically transparent conducting layers: Performance of ring element arrays," Microwave Opt. Technol. Lett., vol. 16, no. 2, pp. 61-63, 1997.

[53] G. I. Kiani, L. G. Olsson, A. Karlsson, K. P. Esselle, and M. Nilsson, "Cross-Dipole Bandpass Frequency Selective Surface for EnergySaving Glass Used in Buildings," IEEE Trans. Antennas Propag., vol. 59, no. 2, pp. 520-525, 2011.

[54] M. Gustafsson, A. Karlsson, A. P. P. Rebelo, and B. Widenberg, "Design of frequency selective windows for improved indoor outdoor communication," IEEE Trans. Antennas Propag., vol. 54, no. 6, pp. 1897-1900, 2006.

[55] R. B. Green et al., "Optically transparent antennas and filters: A smart city concept to alleviate infrastructure and network capacity challenges," IEEE Antennas Propag. Mag., vol. 61, no. 3, pp. 3747, 2019.

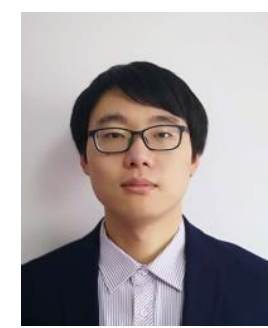

Yiming Yang was born in Shandong Province, China, in 1997. He is an undergraduate student and currently pursuing his B.S. degree in electronic science and technology in University of Electronic Science and Technology of China, Chengdu, China. He is also a visiting student in the integrated microwaves packaging antennas and circuits technology (IMPACT) Lab in King Abdullah University of Science and Technology, Thuwal, Saudi Arabia.

His research interests include design and synthesis of frequency selective surfaces, microwave passive devices, and additive manufacturing of antennas and RF circuits.

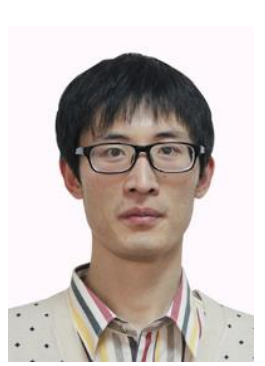

Weiwei Li was born in Henan Province, China, in 1986. He received the B.S. degree in packaging engineering, and M.S. degree in material physics and chemistry from Beijing Institute of Graphic and Communication, Beijing, China, in 2010 and 2013, respectively, and the Ph.D. degree in nanoscience and technology from National Center for Nanoscience and Technology, Chinese Academy of Sciences, Beijing, China, in 2017.

Currently, he is working as a post-doctoral fellow at King Abdullah University of Science and Technology, Thuwal, Saudi Arabia. His research interests include synthesis of functional materials, printed electronics, and radio frequency devices.

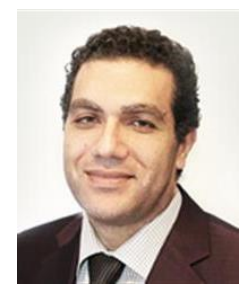

Khaled N. Salama received his masters and doctorate degrees from the Electrical Engineering Department at Stanford University, Stanford, CA, in 2000 and 2005, respectively..

From 2005 to 2009, he was an assistant professor at Rensselaer Polytechnic Institute (RPI), Troy, NY, USA. He is a Professor and founding chair member in the Electrical engineering department at King Abdullah University of Science and Technology. He is currently the director of the sensor initiative at KAUST. He is the co-author of 200 papers and 14 patents on sensors. His research interests cover a variety of interdisciplinary aspects of electronic circuit design and semiconductors fabrication.

Dr. Salama was the recipient of the Defense Advanced Research Projects Agency (DARPA) Stanford-Berkeley Innovators Challenge Award in biological sciences, the work of which was acquired by Lumina Inc. in 2008. His work on CMOS sensors for molecular detection was awarded the Stanford-Berkeley Innovators Challenge Award in biological sciences and was acquired by Lumina Inc in 2008. 


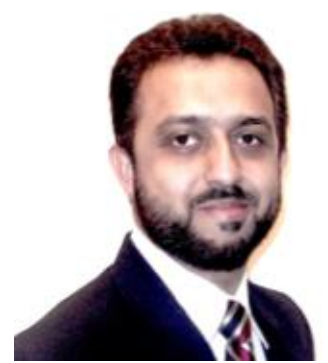

Atif Shamim (SM'13) received his M.S. and $\mathrm{PhD}$ degrees in electrical engineering from Carleton University, Canada in 2004 and 2009 respectively. He was an NSERC Alexander Graham Bell Graduate scholar at Carleton University from 2007 till 2009 and an NSERC postdoctoral Fellow in 2009-2010 at Royal Military College Canada and KAUST. In August 2010, he joined the Electrical Engineering Program at KAUST, where he is currently an Associate Professor and principal investigator of IMPACT Lab. He was an invited researcher at the VTT Micro-Modules Research Center (Oulu, Finland) in 2006. He is an author/co-author of 250 international publications, an inventor on 25 patents and has given over 50 invited talks at various international forums. His research interests are in innovative antenna designs and their integration strategies with circuits and sensors for flexible and wearable wireless sensing systems through a combination of CMOS and additive manufacturing technologies. $\mathrm{He}$ is a Senior Member of IEEE and serves on the editorial board of IEEE Transactions on Antennas and Propagation.

Dr. Shamim's research work has won best paper awards in IEEE EuWiT 2008, IEEE IMS 2016, IEEE MECAP 2016, first prize in IEEE IMS 2019 3MT competition and honorable mention prizes in IEEE APS 2005, IEEE IWAT 2006, IEEE IMS 2014, IEEE IMS 2017 (3MT competition). He was given the Ottawa Centre of Research Innovation (OCRI) Researcher of the Year Award in 2008 in Canada. His work on Wireless Dosimeter won the ITAC SMC Award at Canadian Microelectronics Corporation TEXPO in 2007. Prof. Shamim also won numerous business-related awards, including 1st prize in Canada's national business plan competition and was awarded OCRI Entrepreneur of the year award in 2010. 